\title{
Immune Checkpoint Inhibitors in Small Cell Lung Cancer: A Partially Realized Potential
}

\author{
Samantha A. Armstrong · Stephen V. Liu
}

Received: April 23, 2019 / Published online: June 17, 2019

(c) The Author(s) 2019 approval of atezolizumab as part of first-line therapy for advanced SCLC. While these landmark approvals offer promising novel treatment options for this recalcitrant disease, more work is needed to optimize their delivery and to build upon these important advances.

Keywords: Atezolizumab; Checkpoint inhibitors; Immunotherapy; Nivolumab; SCLC

\section{INTRODUCTION}

Small cell lung cancer (SCLC) is a highly lethal subtype of lung cancer. It is characterized by a rapid onset, an aggressive course, and a uniquely predictable response pattern. Initially, SCLC is highly responsive to chemotherapy; even monotherapy with numerous agents can induce a response [1]. Unfortunately, relapse is just as predictable as response, and in contrast to treatment-naïve SCLC, relapsed SCLC is highly refractory to most agents.

Standard initial therapy for advanced, extensive-stage (ES) SCLC is platinum-based chemotherapy, typically cisplatin or carboplatin combined with etoposide or irinotecan [2]. The response rate is high (51-67\%) but responses are transient, with progression free survival (PFS) typically limited to 4-5.5 months [3]. Survival remains about 10 months or shorter in most series. Despite these poor 
outcomes, treatment has been relatively static for decades, with dozens of phase III trials failing to improve survival. Progress has been elusive as SCLC is a challenging disease to properly study. Fortunately, advances in immunotherapy, specifically implementation of checkpoint inhibitors, have finally changed the treatment landscape for SCLC.

This article is based on previously conducted studies and does not contain any studies with human participants or animals performed by any of the authors.

\section{IMMUNOTHERAPY RATIONALE}

As checkpoint inhibitors began to show promising activity in melanoma and non-small cell lung cancer (NSCLC), their application to SCLC was highly anticipated. SCLC seemed poised to be a highly immune-responsive tumor. A consistent predictor of immune-mediated antitumor response has been a high number of somatic tumor mutations. Among patients with NSCLC treated with pembrolizumab, those with a higher tumor mutational burden (TMB) were more likely to respond to therapy [4]. Tumors with the highest rates of mutations per megabase include melanoma, NSCLC, and bladder cancer-all tumors with responses to immunotherapy [5]. SCLC is a carcinogen-associated tumor and has among the highest rates of mutations per megabase $[5,6]$, which generated enthusiasm for an immunotherapy approach in SCLC.

Furthermore, there is already a strong relationship between SCLC and the immune system. SCLC, perhaps more than nearly any other cancer, is associated with neurologic paraneoplastic syndromes. Host antibodies recognizing a mal-expressed neuronal antigen on the tumor interact with normal host cells causing a litany of potentially disabling symptoms [7]. Many series have reported that the presence of these syndromes is associated with a better cancer prognosis. Lambert-Eaton syndrome, which occurs in $2-3 \%$ of SCLC patients, is associated with improved prognosis, with a median survival of 17.3 months compared to 10 months in patients without Lambert-Eaton [8]. Longer survival has also been associated with SCLC patients affected by anti-Yo cerebellar syndrome [9]. Furthermore, some patients with idiopathic anti-Hu encephalomyelitis or sensory neuropathy were found to have small SCLC lesions only noted at autopsy [10]. These observations suggest that immune-mediated neurologic syndromes may be associated with immunemediated anti-tumor responses, responses that could perhaps be induced with checkpoint inhibitors.

\section{IMMUNOTHERAPY FOR RELAPSED SCLC}

Despite the high anticipation of success with immunotherapy, outcomes have been modest. Pembrolizumab, an anti-PD-1 antibody, has been explored in SCLC in two notable studies. KEYNOTE-028 was a phase Ib basket study that included 24 patients with relapsed ES-SCLC whose tumor expressed PD-L1 in at least $1 \%$ of cells by immunohistochemistry [11]. In this cohort, the response rate was a promising 33\% with a duration of response of 19.4 months. Overall median PFS, though, was only 1.9 months. A larger phase II study included 107 patients with previously treated SCLC, unselected for PD-L1 expression [12]. The response rate was $18.7 \%$ in this larger study; median PFS was still only 2.0 months and median survival was 8.7 months. A pooled analysis of these two studies reported a response rate of $19.3 \%$, a median PFS of 2.0 months [13], and a median survival of 7.7 months. While pembrolizumab clearly had activity in relapsed SCLC, benefit was limited to a minority of patients.

Perhaps the most experience in this setting has been with the anti-PD-1 antibody nivolumab, alone or with the anti-CTLA-4 antibody ipilimumab. Checkmate-032 was a phase I/II study that initially included a non-randomized cohort of patients with relapsed SCLC. Patients were treated with nivolumab alone or in combination with ipilimumab, employing various dosing schedules [14]. Nivolumab alone had a response rate of $10 \%$ with a median PFS of 1.4 months. The addition of ipilimumab 
increased the response rate to $19-23 \%$ but also increased the rate of grade 3 or higher adverse events. PFS was still limited to only 1.4-2.3 months with the combination. Survival was 4.4-7.7 months across these cohorts. A randomized cohort was then added, with 242 patients randomized $3: 2$ to nivolumab monotherapy or in combination with ipilimumab. Response patterns were similar: 12\% with monotherapy and $21 \%$ with the combination [15]. Outcomes were also reported for the 109 patients treated with nivolumab monotherapy in the third-line setting [16]. With a median follow-up of 28.3 months, the response rate was $11.9 \%$ with a median duration of response of 17.9 months. On the basis of these data, nivolumab was granted accelerated approval by the Food and Drug Administration (FDA) on August 16, 2018 for the third-line treatment of advanced SCLC.

Unfortunately, most patients are not eligible for third-line therapy. In an analysis of 432 patients with ES-SCLC treated in Germany, only $22 \%$ of patients received a third line of treatment [17]. Earlier efforts are certainly appealing. Attempts to introduce immunotherapy in the second-line setting, however, have been disappointing. Checkmate 331 was a randomized phase III trial in patients with SCLC that progressed on or relapsed after platinum-based therapy [18]. In this study, 568 patients were randomized $1: 1$ to receive nivolumab monotherapy or investigator's choice between topotecan and amrubicin. Nivolumab did not improve survival, with a median OS of 7.5 months compared to 8.4 months with chemotherapy. Both response rate $(13.7 \%$ vs. $16.5 \%)$ and PFS (1.4 months vs. 3.8 months) numerically favored chemotherapy. A non-comparative, randomized phase II study of atezolizumab monotherapy was also disappointing [19]. Among the 43 eligible patients treated with atezolizumab, there was only one response (2.3\%) with a median PFS of 1.4 months and median survival of 9.5 months.

\section{MAINTENANCE IMMUNOTHERAPY}

Immunotherapy has clear activity in SCLC but failed to improve outcomes compared to standard second line therapy, despite a seemingly low bar. As SCLC becomes significantly more refractory to therapy at relapse, earlier introduction was explored with maintenance strategies. These have been similarly disappointing. A single-arm phase II trial exploring maintenance pembrolizumab included 45 patients with ES-SCLC who had a response or stable disease after first line platinum-etoposide chemotherapy [20]. The target PFS was 3.0 months, based on historical standards. The observed PFS was only 1.4 months.

A larger, phase III maintenance study reported similar results. Checkmate- 451 included 834 patients with ES-SCLC who had an ongoing response or stable disease after four cycles of platinum-based chemotherapy [21]. Patients were randomized 1:1:1 to receive nivolumab $1 \mathrm{mg} / \mathrm{kg}$ plus ipilimumab $3 \mathrm{mg} / \mathrm{kg}$ every 3 weeks (with maintenance nivolumab $240 \mathrm{mg}$ every 2 weeks after four cycles of induction therapy), nivolumab $240 \mathrm{mg}$ every 2 weeks as monotherapy or placebo. The primary endpoint was an improvement in survival with nivolumab plus ipilimumab as compared to placebo. This endpoint was not met. The OS HR was 0.92 (95\% CI 0.8-1.1) with a median OS of 9.2 months with nivolumab plus ipilimumab and 9.6 months with placebo. PFS was marginally improved at 1.7 months vs. 1.4 months (HR $0.72,95 \%$ CI $0.60-0.87$ ) but toxicity was significantly worse, with treatment-related adverse events leading to discontinuation in $29 \%$ of patients, compared to $<1 \%$ with placebo. Comparisons of nivolumab monotherapy to chemotherapy were exploratory, given the hierarchical analysis, but provided similar results. Nivolumab had a median OS of 10.4 months compared to 9.6 with placebo (HR $0.84,95 \%$ CI 0.7-1.0) with a PFS of 1.9 months vs. 1.4 months (HR $0.67,95 \%$ CI 0.56-0.81).

\section{FIRST-LINE IMMUNOTHERAPY STRATEGIES}

While second-line and maintenance efforts have been disappointing, the greatest potential for impact has always been as first-line therapy. SCLC has a very high rate of attrition with fewer 
patients eligible for therapy in later lines. Treatment-naïve patients also lack the cumulative toxicity of prior therapy, and the impact of this prior therapy on the likelihood of generating an immune response is not clear. Given the aggressive natural history of SCLC, however, and the relatively low response rates previously noted, immunotherapy alone carried too great a risk in an unselected population. If response was not seen, patients would likely forfeit the reliable, though admittedly transient, benefit of chemotherapy. This was one reason why combinations of chemotherapy and immunotherapy were explored as first-line therapy (Table 1). Chemotherapy can provide a reliable initial benefit and the immediate addition of immunotherapy could potentially improve long-term outcomes. There is also a potential synergy with chemotherapy-immunotherapy combinations. Chemotherapy can impact myeloid-derived suppressor cells and facilitate tumor antigen release; chemotherapy is a known immune modulator and its effects may promote an immune response [22].

\section{FIRST-LINE ANTI-CTLA-4}

The first combination efforts were with chemotherapy and anti-CTLA-4 therapy and were decidedly negative. The addition of ipilimumab to carboplatin plus paclitaxel, as a firstline therapy for ES SCLC, showed no improvement in PFS (3.9 months with ipilimumab vs. 5.2 months with placebo) or overall survival
(9.1 months vs. 9.9 months) [23]. A phase III trial of platinum plus etoposide with ipilimumab or placebo was also negative [24]. This study included 954 patients and ipilimumab compared to placebo did not improve survival (11.0 months vs. 10.9 months), PFS (4.6 months vs 4.4 months), or response rate (62\% in both arms). It did increase toxicity, with treatmentrelated discontinuation noted in $18 \%$ of patients compared to $2 \%$ with placebo.

\section{FIRST-LINE ANTI-PD-L1}

Fortunately, survival was improved with the addition of the anti-PD-L1 antibody atezolizumab to chemotherapy. IMpower 133 was a global, randomized, placebo-controlled, double blind phase I/III trial that included 403 patients with treatment-naïve ES-SCLC [25]. All patients received four cycles of carboplatin AUC 5 on day 1 with etoposide $100 \mathrm{mg} / \mathrm{m}^{2}$ on days 1-3 and were randomized to receive concurrent atezolizumab $1200 \mathrm{mg}$ on day 1 or placebo, followed by maintenance atezolizumab or placebo. The co-primary endpoints were overall survival and investigator-assessed PFS; IMpower 133 met both of its primary endpoints.

The addition of atezolizumab improved overall survival, with an improvement in median OS from 10.3 months to 12.3 months and an HR for death of 0.70 (95\% CI 0.54-0.91). Atezolizumab improved the 1-year survival rate from $38.2 \%$ to $51.7 \%$. PFS was also superior with atezolizumab, with an HR of 0.77 (95\% CI

Table 1 Select randomized trials featuring concurrent chemotherapy and immunotherapy

\begin{tabular}{|c|c|c|c|c|c|c|}
\hline Study & Chemotherapy & Intervention & $\begin{array}{l}\text { PFS } \\
\text { (months) }\end{array}$ & PFS HR & $\begin{array}{l}\text { OS } \\
\text { (months) }\end{array}$ & OS HR \\
\hline \multirow{2}{*}{$\begin{array}{l}\text { Reck et al. } \\
\text { [23] }\end{array}$} & \multirow{2}{*}{$\begin{array}{l}\text { Carboplatin plus } \\
\text { paclitaxel }\end{array}$} & Ipilimumab & 3.9 & \multirow[t]{2}{*}{$0.93(059-1.48)$} & 9.1 & \multirow[t]{2}{*}{$0.89(0.57-1.39)$} \\
\hline & & Placebo & 5.2 & & 10.5 & \\
\hline \multirow{2}{*}{$\begin{array}{l}\text { Reck et al. } \\
{[24]}\end{array}$} & \multirow{2}{*}{$\begin{array}{l}\text { Platinum plus } \\
\text { etoposide }\end{array}$} & Ipilimumab & 4.6 & \multirow[t]{2}{*}{$0.85(0.75-0.97)$} & 11.0 & \multirow[t]{2}{*}{$0.94(0.81-1.09)$} \\
\hline & & Placebo & 4.4 & & 10.9 & \\
\hline \multirow{2}{*}{$\begin{array}{l}\text { Horn et al. } \\
\text { [25] }\end{array}$} & \multirow{2}{*}{$\begin{array}{l}\text { Carboplatin plus } \\
\text { etoposide }\end{array}$} & Atezolizumab & 5.2 & \multirow[t]{2}{*}{$0.77(0.62-0.96)$} & 12.3 & \multirow[t]{2}{*}{$0.70(0.54-0.91)$} \\
\hline & & Placebo & 4.3 & & 10.3 & \\
\hline
\end{tabular}


0.63-0.96). There was no difference in response rate and no new safety signals were identified. Patients in both arms received a median of four doses of carboplatin and 12 doses of etoposide, suggesting that the addition of atezolizumab did not compromise the ability to deliver four full cycles of chemotherapy. These landmark results represent the first improvement in survival in several decades, establishing atezolizumab plus carboplatin plus etoposide as the new standard of care in ES-SCLC. The IMpower 133 regimen was approved by the FDA as first-line therapy for ES-SCLC on March 18, 2019. Two other large phase III SCLC trials have completed accrual with results pending. KEYNOTE 604 randomized patients to chemotherapy alone or with pembrolizumab (NCT03066778) and CASPIAN features three arms: chemotherapy alone, chemotherapy with the anti-PD-L1 antibody durvalumab, or chemotherapy with durvalumab and the CTLA4 inhibitor tremelimumab (NCT03043872).

\section{BIOMARKERS}

In previously treated patients, there is an impressive duration of response to checkpoint inhibitors that is balanced by a very short median PFS. This suggests that most of the benefit is carried by a small subset of patients. Identification of that subset is critical to optimize therapy-not only to ensure patients receive the proper therapy but also to understand why most patients do not respond. The search for a predictive biomarker has been challenging. PD-L1 expression has been explored. In KEYNOTE-158, expression of PD-L1 on either tumor or stromal cells was explored and tumors with expression had superior outcomes [12]. Response in PD-L1-positive tumors (using this combined proportion score, CPS) was $35.7 \%$ vs. $6 \%$ in the PD-L1-negative tumors. This also translated to a superior survival of 14.9 months vs. 5.9 months and a 1 -year survival rate of $53.1 \%$ vs. $30.7 \%$. This pattern was not seen in the Checkmate-032 study, where patients with PD-L1 expression had an inferior response rate with nivolumab monotherapy $(9 \%$ vs. $14 \%)$ or in combination with ipilimumab $3 \mathrm{mg} / \mathrm{kg}$ (10\% vs. 32\%) [15]. In Checkmate-032, however, TMB was associated with a higher response rate. With nivolumab alone, the response rate in patients with high TMB was $21.3 \%$ compared to $4.8 \%$ in patients with low TMB [26]. With nivolumab and ipilimumab, responses were noted in $46.2 \%$ of patients with high $\mathrm{TMB}$ and $22.2 \%$ with low TMB. However, blood-based TMB, explored in IMpower 133, was not associated with a difference in outcome, with patients above and below pre-specified thresholds all favoring the addition of atezolizumab [24].

\section{CONCLUSION}

Small cell lung cancer is a recalcitrant, unforgiving disease whose course can only be minimally altered by our current interventions. Chemotherapy has provided reliable initial responses for decades, but its transient nature underscores the need for newer treatment strategies. Immunotherapy has finally improved outcomes. While nivolumab is an approved option in the third-line space, the failures of immunotherapy in the second-line and maintenance settings have been disappointing. Fortunately, outlook is now buoyed by the success of IMpower 133. The addition of concurrent atezolizumab to carboplatin and etoposide has finally delivered on improving survival, offering patients a long-awaited novel approach. We now look to the field to build upon this success, confident that the next advance will not take decades to materialize.

\section{ACKNOWLEDGEMENTS}

Funding. No funding or sponsorship was received for publication of this article.

Authorship. All named authors meet the International Committee of Medical Journal Editors (ICMJE) criteria for authorship for this article, take responsibility for the integrity of the work as a whole, and have given their approval for this version to be published. 
Disclosures. SAA reports no relevant conflicts. SVL reports serving as a paid consultant and/or advisory board member for Apollomics, AstraZeneca, Boehringer Ingelheim, BristolMyers Squibb, Celgene, G1 Therapeutics, Genentech/Roche, Heron, Ignyta, Inivata, Janssen, Lilly, Merck, Pfizer, Regeneron, Taiho (DSMB), Takeda/Ariad, and Tempus. SVL has received research funding from AstraZeneca, Bayer, Blueprint, Bristol-Myers Squibb, Clovis, Corvus, Esanex, Genentech/Roche, Ignyta, Lilly, Lycera, Merck, Molecular Partners, OncoMed, Pfizer, Rain Therapeutics, and Threshold.

Compliance with Ethics Guidelines. This article is based on previously conducted studies and does not contain any studies with human participants or animals performed by any of the authors.

Open Access. This article is distributed under the terms of the Creative Commons Attribution-NonCommercial 4.0 International License (http://creativecommons.org/licenses/ by-nc/4.0/), which permits any noncommercial use, distribution, and reproduction in any medium, provided you give appropriate credit to the original author(s) and the source, provide a link to the Creative Commons license, and indicate if changes were made.

\section{REFERENCES}

1. Shepherd FA. The role of chemotherapy in the treatment of small cell lung cancer. Chest Surg Clin N Am. 1997;7(1):113-33.

2. Rossi A, Di Maio M, Chiodini P, et al. Carboplatinor cisplatin-based chemotherapy in first-line treatment of small-cell lung cancer: the COCIS metaanalysis of individual patient data. J Clin Oncol. 2012;30(14):1692-8.

3. Roth BJ, Johnson DH, Einhorn LH, et al. Randomized study of cyclophosphamide, doxorubicin, and vincristine versus etoposide and cisplatin versus alternation of these two regimens in extensive small-cell lung cancer: a phase III trial of the Southeastern Cancer Study Group. J Clin Oncol. 1992;10(2):282-91.
4. Rizvi NA, Hellmann MD, Snyder A, et al. Cancer immunology. Mutational landscape determines sensitivity to PD-1 blockade in non-small cell lung cancer. Science. 2015;348(6230):124-8.

5. Alexandrov LB, Nik-Zainal S, Wedge DC, et al. Signatures of mutational processes in human cancer. Nature. 2013;500(7463):415-21.

6. Peifer M, Fernandez-Cuesta L, Sos ML, et al. Integrative genome analyses identify key somatic driver mutations of small-cell lung cancer. Nat Genet. 2012;44(10):1104-10.

7. Kanaji N, Watanabe N, Kita N, et al. Paraneoplastic syndromes associated with lung cancer. World J Clin Oncol. 2014;5(3):197-223.

8. Maddison P, Newsom-Davis J, Mills KR, Souhami RL. Favourable prognosis in Lambert-Eaton myasthenic syndrome and small-cell lung carcinoma. Lancet. 1999;353(9147):117-8.

9. Rojas I, Graus F, Keime-Guibert F, et al. Long-term clinical outcome of paraneoplastic cerebellar degeneration and anti-Yo antibodies. Neurology. 2000;55(5):713-5.

10. Dalmau J, Graus F, Rosenblum MK, Posner JB. AntiHu-associated paraneoplastic encephalomyelitis/ sensory neuronopathy. A clinical study of 71 patients. Medicine (Baltimore). 1992;71(2):59-72.

11. Ott PA, Elez E, Hiret $\mathrm{S}$, et al. Pembrolizumab in patients with extensive-stage small-cell lung cancer: results from the phase Ib KEYNOTE-028 study. J Clin Oncol. 2017;35(34):3823-9.

12. Chung HC, Lopez-Martin JA, Kao SC, et al. Phase 2 study of pembrolizumab in advanced small-cell lung cancer (SCLC): KEYNOTE-158. J Clin Oncol. 2018;36(15_suppl):8506.

13. Chung HC, Piha-Paul SA, Lopez-Martin JA, et al. Pembrolizumab after two or more lines of prior therapy in patients with advanced SCLC: results from the KEYNOTE-028 and KEYNOTE-158 studies. In: Proceedings of the 110th annual meeting of the American Association for Cancer Research, 2019. 2019 Mar 29-Apr 3; Atlanta, GA. Philadelphia (PA): AACR; 2019 (Abstract CT073).

14. Antonia SJ, Bendell JC, Taylor MH, et al. Phase I/II study of nivolumab with or without ipilimumab for treatment of recurrent small cell lung cancer (SCLC): CA209-032. J Clin Oncol. 2015;33:abstr 7503.

15. Hellmann MD, Ott PA, Zugazagoitia J, et al. Nivolumab (nivo) \pm ipilimumab (ipi) in advanced smallcell lung cancer (SCLC): first report of a randomized 
expansion cohort from CheckMate 032. J Clin Oncol. 2017;35(15_suppl):8503.

16. Ready N, Farago AF, de Braud F, et al. Third-line nivolumab monotherapy in recurrent SCLC: CheckMate 032. J Thorac Oncol. 2018;14:237-44.

17. Steffens CC, Elender C, Hutzschenreuter U, et al. Treatment and outcome of 432 patients with extensive-stage small cell lung cancer in first, second and third line-results from the prospective German TLK cohort study. Lung Cancer. 2019;130:216-25.

18. Reck M, Vicente D, Ciuleanu T, et al. Efficacy and safety of nivolumab (nivo) monotherapy versus chemotherapy (chemo) in recurrent small cell lung cancer (SCLC): results from CheckMate 331. Ann Oncol. 2018;29(10):LBA5x39-43.

19. Pujol JL, Greillier L, Audigier-Valette C, et al. A randomized non-comparative phase 2 study of antiprogrammed cell death-ligand 1 atezolizumab or chemotherapy as second-line therapy in patients with small cell lung cancer: results from the IFCT1603 trial. J Thorac Oncol. 2019;14(5):903-13.

20. Gadgeel SM, Pennell NA, Fidler MJ, et al. Phase II study of maintenance pembrolizumab in patients with extensive-stage small cell lung cancer (SCLC). J Thorac Oncol. 2018;13(9):1393-9.

21. Owonikoko T, Kim HR, Govindan R, et al. Nivolumab (nivo) plus ipilimumab (ipi), nivo, or placebo (pbo) as maintenance therapy in patients (pts) with extensive disease small cell lung cancer (ED-SCLC) after first-line (1L) platinum-based chemotherapy (chemo): results from the double-blind, randomized phase III CheckMate 451 study. Ann Oncol. 2019;30(Supplement_2):LBA1_PR.

22. Destefano CB, Liu SV. Combinatorial immunotherapy and chemotherapy. In: Patel SP, Kurzrock R, editors. Early phase cancer immunotherapy. Cham: Springer; 2018. p. 199-218.

23. Reck M, Bondarenko I, Luft A, et al. Ipilimumab in combination with paclitaxel and carboplatin as first-line therapy in extensive-disease-small-cell lung cancer: results from a randomized, doubleblind, multicenter phase 2 trial. Ann Oncol. 2013;24(1):75-83.

24. Reck M, Luft A, Szczesna A, et al. Phase III randomized trial of ipilimumab plus etoposide and platinum versus placebo plus etoposide and platinum in extensive-stage small-cell lung cancer. J Clin Oncol. 2016;34(31):3740-8.

25. Horn L, Mansfield AS, Szczesna A, et al. First-line atezolizumab plus chemotherapy in extensive-stage small-cell lung cancer. $N$ Engl J Med. 2018;379:2220-9.

26. Hellmann MD, Callahan MK, Awad MM, et al. Tumor mutational burden and efficacy of nivolumab monotherapy and in combination with ipilimumab in small-cell lung cancer. Cancer Cell. 2018;33(5):853e4-861e4. 